\title{
The Magnitude of Pre-lacteal Feeding Practice and its Influencing Factors Among Mothers with Children Under the Age of Two Years in Afar Region of Ethiopia: A Community based Quantitative and Qualitative Study
}

Temesgen Gebeyehu ( $\nabla$ tomigeb2006@gmail.com )

Samara University

Abel Gebru

Samara University

Nigus Agegnehu

Samara University

Research Article

Keywords: Pre-lacteal feeding, factors, mother, children

Posted Date: October 12th, 2021

DOl: https://doi.org/10.21203/rs.3.rs-944815/v1

License: (c) (1) This work is licensed under a Creative Commons Attribution 4.0 International License.

Read Full License 
1 The magnitude of pre-lacteal feeding practice and its influencing

2 factors among mothers with children under the age of two years in

3 Afar region of Ethiopia: A community based quantitative and 4 qualitative study.

5 Temesgen Gebeyehu Wondmeneh ${ }^{1 *}$, Abel Gebru ${ }^{2}$, Nigus Agegnehu ${ }^{3}$

6 1. Department of Public health (MPH in epidemiology), college of health science, Samara University, Ethiopia

2. Department of Public health (MPH in nutrition), college of health science, Samara University, Ethiopia

3. Department of Public health(BSc Public health officer), college of health science, Samara University, Ethiopia

${ }^{*}$ Correspondence author

Temesgen Gebeyehu

Email:tomigeb2006@gmail.com

16 
Background: Pre-lacteal feeding is a barrier to implement optimal breastfeeding practices and caused new-born disease. Pre-lacteal feeding is primarily practiced in developing countries, where cultural and social structures are based on descent and relationships. The aim of study was to determine the magnitude of pre-lacteal feeding practice and its influencing factors among mothers with children under the age of 2-years in Afar region of Ethiopia.

Method: A community based cross sectional study supplemented by qualitative study was employed. In the quantitative study, 235 study participants were included, whereas seventeen study subjects participated in the qualitative study. Bivariate and multivariate logistic regression was used to determine factors associated with prelacteal feeding practice. The qualitative data was analyzed using thematic approach.

Result: The magnitude of pre-lacteal feeding was $36.6 \%$. Being a housewife (AOR=4.1, 95\% Cl: 1.4-12.2) and having male indexed child (AOR=4.9, 95\% Cl: 1.813.5) were more likely to practice pre-lacteal feeding than those of trade and female, respectively. However, pre-lacteal feeding practice decreased in those mothers with three and above ante natal care visits (AOR=0.31, 95\% Cl: $0.1-0.9)$, delivered at health institutes (AOR=0.1, 95\% Cl: 0.02-0.3), starting breastfeeding within one hour $(\mathrm{AOR}=0.04$, at $95 \% \mathrm{Cl}: 0.01-0.1)$ and getting breast feeding counseling $(\mathrm{AOR}=0.21$, at $95 \% \mathrm{Cl}: 0.06-0.7)$. Milk, plain water, sugar solution, honey, butter, dates ('temir'), a leaf, and condo pepper were the most common pre-lacteal foods reported by study participants. Cultural beliefs and the assumption of cleaning new-born throat and bowel were the most common reason to practice pre-lacteal feeding. 
48 Conclusion: The magnitude of pre-lacteal feeding was high due to socio-cultural

49 and maternal health service utilization factors. Water, milk products, sweet foods and

50 some of plant types were the most common pre-lacteal foods.

51 Key words: Pre-lacteal feeding, factors, mother, children 
Breast-feeding delay was frequently a cause of pre-lacteal feeding. Cultural taboos and beliefs influenced the majority of mothers' weaning (1). The practice of giving certain types of food to babies before commencing breast milk is common in both urban and rural areas. Some of the most frequently reported pre-lacteal meals are tamarind juice, honey, jaggery, sugar and glucose water, animal milk, and commercial powdered milk (2). From 2010 to 2018, the pooled prevalence of prelacteal feeding in eleven East African countries was 12\%, with the greatest rate $(39 \%)$ in the Comoros and the lowest in Malawi (3\%). Pre-lacteal feeding was associated with wealth index, antenatal care (ANC) visit, health institutional delivery and mode of delivery (3). In a meta-analysis study, the pooled prevalence of prelacteal feeding was found to be $25.29 \%$ in Ethiopia. Antenatal care, infant feeding counseling, timely breastfeeding initiation and urban residence were associated with a lower risk of pre-lacteal feeding, but home birth was associated with a greater risk (4). In Sodo district of southern Ethiopia, the prevalence of pre-lacteal feeding was $20.6 \%$. Plain water $(7.7 \%)$ was the most common pre-lacteal feeding and the main reason was lack of breast milk (6.5\%). Lack of breastfeeding advice and the avoidance of colostrum were statistically significant with pre-lacteal feeding practice (5). Pre-lacteal feeding was practiced by $12.6 \%$ of women in southern Ethiopia. Lack of mother education, colostrum avoidance, home delivery, the absence of information regarding the risk of pre-lacteal feeding and unawareness about breast feeding practices were associated with pre-lacteal feeding (6). Pre-lacteal feeding was found to be $46.4 \%$ in south-west Ethiopia. Breastfeeding after one hour of delivery, giving birth at home, failing to recognize the hazards of pre-lacteal feeding, and believing in the benefits of pre-lacteal feeding were identified as key risk factors 
91 in pre-lacteal feeding practices (7). The prevalence of pre-lacteal feeding was $10.1 \%$

in the Axum town, North Ethiopian. Mothers with no previous children, birth spacing of less than two years, not giving colostrum, less than four anti-natal care visits, cesarean section and perceiving the benefits of pre-lacteal feeding were associated with pre-lacteal feeding (8). In a study conducted in Eastern Tigray region of Ethiopia, $24.7 \%$ of women practiced pre-lacteal feeding. Pre-lacteal feeding had associated to parity, late breastfeeding initiation and colostrum avoidance (9). Prelacteal feeding decreased from $29 \%$ in 2005 to $8 \%$ in 2016 . The practice of prelacteal feeding was significantly influenced by distance from the health institution, wealth level, occupation, antenatal care visits and desired last child. Lower rates of pre-lacteal feeding were seen in Benishangul, Tigray, most of Amhara, the western part of Gambela and the eastern parts of the southern nation nationality people region. The Somalia and Afar regions had greater rates of pre-lacteal feeding (10). A meta-analysis was conducted in Ethiopia to investigate the relationship between home delivery and pre-lacteal feeding. Pre-lacteal feeding accounted for a total of 26.95\% (95\% Cl: $6.1 \%-75.8 \%)$. The highest prevalence was in the Afar area. The pooled odds of pre-lacteal feeding was increased by 5.16 times when comparing women who gave birth at home to those who gave in health institutions (95\% Cl: 3.77.2) (11). In February 2013, a cross-sectional study was conducted in public health institution in the Harare district in Eastern Ethiopia. About $45.4 \%$ mothers gave prelacteal fluid to their babies. The most common pre-lacteal diets were sugar water and milk, which accounts for $43.5 \%$ and $25.1 \%$, respectively. Home delivery, failure to attend antenatal care (ANC), late breastfeeding initiation and friend influence were statistically predictors of pre-lacteal feeding (12). In 2016, Zone four districts of Afar region in Ethiopia, a community-based cross-sectional study was conducted. About 
$42.9 \%$ of mothers gave their children pre-lacteal feeding. Raw butter $(54.2 \%)$ and

117 animal milk (28.5\%) were the most frequent pre-lacteal feeding. Pre-lacteal feeding was significantly associated with housewife, having a husband with no formal education, starting breastfeeding after one hour of birth, having a higher household income and a lack of information about good breastfeeding practice (13). A crosssectional study was conducted in the town of Samara-Logia in 2017 . Ninety percent of mothers responded that breastfeeding is beneficial to their infants. However, $45.3 \%$ of women thought it was useful to provide feeds other than breast milk. About $45 \%$ of the women started breastfeeding within an hour after birth. Within three days after delivery, more than half of the newborns $(62.5 \%)$ had received pre-lacteal feeding (14). A community-based cross-sectional study was conducted in Dubti district, Afar region. About $93 \%$ of mothers began breast feeding their babies within an hour after birth. Pre-lacteal feeding and avoided colostrum were practiced by $16.8 \%$ and $15.6 \%$ of mothers, respectively (15).

A clear understanding the magnitude of pre-lacteal feeding practice and associated factors is essential to create an appropriate intervention in a pastoral community where the social and cultural conditions were conducive to such practice. The current study explores favorite socio-cultural characteristics and maternal health service utilization factors that were associated with pre-lacteal feeding practice since there is limited study in the Afar region of Ethiopia. Exploring the key factors of prelacteal feeding practice can be achieved by qualitative and quantitative study. The finding could be useful in establishing intervention programs and promoting early breastfeeding initiation as a strategy to enhance mother and new-born health at the community, regional, and national levels, as well as in developing nations. 


\section{Study area}

143 This study was conducted in Algante village and Dasa village which were found around Dubti town in Afar region of Ethiopia, where the majority of the population lives a pastoralist lifestyle. These two districts were chosen purposively because most of the people live permanently in this area due to the existence of the Awash

147 River, which is used for irrigation, grazing and drinking of animals. Dubti town is found in Dubti woreda and located 586 kilometers from Addis Ababa, North-East

149 Ethiopia. The total households in Algante and Dasa villages were 275 and 241, respectively. The Afar region has the lowest latitude in Africa and a hot dry climate condition that forces pastoralist community to move around constantly in search of grazing land and water.

Culture: Majority of population in Afar region follows Muslim religious. The social systems of the Afar are based on descent and relationships. The Afar has a patrilineal descent system that assigns a person to a certain clan (mela). Polygamous marriage is practiced in accordance with Islamic rule. Cross-cousin marriages (in the region called as Absuma) are compulsory. The ability and willingness of a wife for continue delivering in her parents' home after marriage may be depending on her husband's financial and social support. Afar peoples have a proclivity towards having as many children as possible. Men are the heads of their respective families in Afar households. Men are often seen as authoritative figures and hold the greater share of property and child custody rights. Despite the fact that domestic decision-making is frequently dependent on subsequent talks with women, men have the final word. Although livestock can be owned separately, they are 
considered household assets. Children have their own herds of animals. An infant is given female goats or a camel after birth to "look the luck." This happens during initiation of ceremonies like circumcision or umbilical cord tied. The child is considered lucky when the animal reproduces and survives in the hazard environment. Being first baby was an advantage of receiving more animals. Female children are usually given fewer animals than male. In Afar society, women have less social status than men. Furthermore, daughters do not inherit property equally with sons when the head of the household dies (16). In the Afar community, female genital mutilation is a common practiced. Women, men, religious leaders and traditional birth attendants are a key role in the continued practice of female genital mutilation (FGM) (17).

\section{Study design and sample size determination}

A community-based cross-sectional study with qualitative study was used to investigate pre-lacteal feeding practice and associated factors. The research conducted from July 18 to July 23,2021 . The sample size for the quantitative study was estimated using $16.8 \%$ prevalence of pre-lacteal feeding in Dubti district (15) and a $95 \%$ confidence level with $5 \%$ precision. Thus, the sample size was 215 . By adding 10\% none response rate, the final sample size was 237 .

\section{Inclusion and exclusion criteria of study population.}

184 Biological mothers with children under the age of two years in the specified villages were included in the study. Non-biological mothers, women who had not lived in the study area for six months, and mothers who were unable to talk or hear were also 
informants for the qualitative study since they were the main agents in the practice of pre-lacteal feeding and had more information about it.

\section{Variables and operational definitions}

191 The independent variables were selected after a thorough review of the literature.

192 The dependent variable was pre-lacteal feeding practice, which was recorded as a binary result (yes/no).

194 Pre-lacteal feeding: Giving any solid or liquid foods other than breast milk during the 195 first three days after delivery.

\section{Sampling method and procedures}

197 Quantitative data: Systematic sampling technique was used to select the required study subjects. The sampling interval $(K)$ was determined by dividing the total number of households in the two villages by the total sample size, which is approximately two. Equal sample size was allocated for each village. To decide which direction (north, south, east, and west) to begin data collection, we used a lottery method. Following that, the first household was chosen by lottery method from the first two households, and then every second household was selected until the required sample size was attained.

Qualitative data: The participants were selected from mothers with children under the age of two years and key informants. Key informants were selected from traditional birth attendants using a purposive sample technique. Key informants were recruited with the help of community elders and community health extension workers. The process of selection was continued till the information was redundant. The interview 
participants for the qualitative study were not the same as those who were sampled

211 for the quantitative data participants.

\section{Data collection instruments, methods and procedures}

213 First the qualitative data was collected by In-depth interviews using open-ended 214 guiding question which was developed based on the study objective. To collect quantitative data, closed-ended structured interview questionnaires that were constructed from literatures were used. Five data collectors were trained to collect quantitative data, and four data collectors were recruited and trained to collect qualitative data. For both qualitative and quantitative data collection, all of the data collectors were BSc degree in public health officer. During qualitative data collection, the two data collectors (one note taker) were allocated to each village at the same time. The purpose of study was explained for each study participant before data collection started. Those who agreed to take part in the study were then interviewed face-to-face in a quiet location at their home in order to better understand each other and ensure confidentiality. Information was collected on women's demographic, socio-cultural and obstetric characteristics as well as pre-lacteal feeding practices. The guideline used to conduct in-depth interviews was shown (Table 1).

227 Table 1: Shows the main theme included in the in-depth interviewing guideline.

\begin{tabular}{ll}
\hline Participants & Theme \\
\hline Mothers & Types of pre-lacteal foods given to the \\
& new-born and the reason to practice \\
& them. \\
& Types of pre-lacteal foods given to the
\end{tabular}


new-born and the reason to practice these foods

\section{Data analysis}

230 The data was entered into Epidata version 3.1 and then exported to SPSS software version 23. The study variables were described using descriptive statistics. Bivariate analysis was used to investigate if there was an association between various independent variables and pre-lacteal feeding practice. Variables with a P-value of less than 0.05 in the bivariate analysis were considered eligible for multivariable analysis, and thus put into a multivariable logistic regression model to account any confounding factors. The odds ratio with a 95\% confidence interval was reported in the result. A p-value of less than 0.05 was declared statistically significant. For qualitative data, the field notes were verbatim transcribed. The transcribed contents were manually read several times, then coded and grouped into categories. Finally, theme was developed. The results of the thematic analysis are presented in the form of narrative with supporting quotes. At the end, the qualitative study's findings were triangulated with the quantitative findings.

\section{Data Quality assurance}

Before the actual data collection, $5 \%$ of the sample size was pre-tested outside the study area and certain questions were amended after pre-testing. Data collectors were chosen based on their ability to speak the local language (native speaker of Afar language) and previous experience with data collection. Questionnaires were prepared in English and then translated to the local language (Afar). In addition, supervisors and the primary investigators double-checked each questionnaire on a 
250 daily basis to ensure there were no errors. Cleaning of missing values was done in

251 SPSS using frequency. The field notes were transcribing verbatim into English by a

252 third person who was native to Afar language and had translation experience.

253 Finally, the investigators double-checked the transcribed data.

\section{Ethical clearance}

255 The Institutional Review Board of Samara University College of Health Science gave 256 ethical clearance. The selected villages received a letter of cooperation from the 257 administration of Dubty woreda health office. Informed consent was obtained from 258 each study participant after explanation of the study's aim and from the legal 259 guardians of patients with no formal education. All methods were performed in 260 accordance with the relevant guidelines and regulations. The right of study subjects 261 to refuse or to participate in the study at any time was respected. Any personal 262 identifiers were eliminated from questionnaire to preserve confidentiality. No one was 263 harmed as a result of taking part in this research. 
272 Socio-demographic characteristic of mothers in Dubti woreda, North East 273 Ethiopia

274 A total of 235 study subjects were recruited, with a response rate of $99.2 \%$. About

275

276

277

278

279

280

281

282

$28.1 \%$ of mothers were in the age group of $26-30$ years. The mean age of children was $1 \pm 5.1$ month with a range of 1-19 months. Seventy-five percent of the women were married. Housewives accounted for $64.3 \%$ of women. The majority of study subjects were Muslim (69\%). Both the mothers and fathers had nearly similar educational status. Most of study participants had a family of five or more members (61.7\%). Thirty-two percent of study participants had average monthly income of less than 2501 birr, while around a similar percentage (32.3\%) of participants had average monthly incomes of more than 4500 birr (Table 2).

Table 2: Socio-demographic characteristic of mothers in Dubti woreda, North East Ethiopia

\begin{tabular}{lll}
\hline Variable & Category & Number (\%) \\
\hline & $=<20$ & $44(18.7)$ \\
& $21-25$ & $34(14.5)$ \\
Mothers Age (in year) & $26-30$ & $66(28.1)$ \\
& $31-35$ & $57(24.3)$ \\
& $=>36$ & $34(14.5)$ \\
the mean age of & $1 \pm 5.1$ month (range:1-19 months) \\
children & & \\
Marital status & married & $177(75.3)$ \\
& unmarried & $58(24.7)$
\end{tabular}




$\begin{array}{lll}\text { Occupation } & \text { Housewife } & 151(64.3) \\ & \text { Trade } & 84(35.7) \\ \text { Religious } & \text { Orthodox } & 73(31.1) \\ & \text { Muslim } & 162(69) \\ \text { Mother education } & \text { No formal education } & 118(50.2) \\ & \text { Formal education } & 117(49.8) \\ \text { Husband education } & \text { No formal education } & 119(52.2) \\ & \text { Formal education } & 109(47.8) \\ \text { Family size } & \text { Below five } & 90(38.3) \\ & \text { Five and above } & 145(61.7) \\ \text { Family income } & 2500 \text { and below } & 74(31.5) \\ \text { (Ethiopian birr) } & 2501-3500 & 51(21.7) \\ & 3501-4500 & 34(14.5) \\ & 4501 \text { and above } & 76(32.3)\end{array}$

288 In this study, $41.7 \%$ of the study subjects had a birth interval of more than two years.

289 From the total of respondents, $28.5 \%$ had first child birth order. More than half of the 290 participants (56.6\%) had male indexed child. Nearly three-quarters of mothers 291 (72.8\%) got three or more antenatal care visits. Almost half of mothers (50.6\%) gave 292 birth at home. Fifty-four percent of mothers initiated breastfeeding within one hour, 293 while $28.9 \%$ started after one day. Breastfeeding counseling was received by half of 294 the study participants (49.8\%) (Table 3). 
295 Table 3: shows study participants' health service utilization and maternal 296 characteristics in Dubti woreda, North East Ethiopia.

\begin{tabular}{|c|c|c|}
\hline Variable & Category & No (\%) \\
\hline \multirow[t]{2}{*}{ Birth interval } & 2-years and below & $70(29.8)$ \\
\hline & Above 2-years & $98(41.7)$ \\
\hline \multirow[t]{2}{*}{ Birth order of indexed child } & First child & $67(28.5)$ \\
\hline & Subsequent child & $168(71.5)$ \\
\hline \multirow[t]{2}{*}{ Gender of indexed child } & Male & $133(56.6)$ \\
\hline & Female & $102(43.4)$ \\
\hline \multirow[t]{2}{*}{ Frequency of antenatal care } & Three and above & $171(72.8)$ \\
\hline & Below three & $64(27.2)$ \\
\hline \multirow[t]{2}{*}{ Place of birth } & Health institution & $116(49.4)$ \\
\hline & Home & $119(50.6)$ \\
\hline \multirow[t]{3}{*}{ Breastfeeding initiation time } & Within one hour & $127(54)$ \\
\hline & Between one hour-one day & $40(17)$ \\
\hline & After one day & $68(28.9)$ \\
\hline \multirow[t]{2}{*}{ Breastfeeding counseling } & Yes & $117(49.8)$ \\
\hline & No & $118(50.2)$ \\
\hline
\end{tabular}

297

298 The magnitude of pre-lacteal feeding and the reasons for practicing these 299 foods in Dubti woreda, North East Ethiopia.

300 The magnitude of pre-lacteal foods practice by those mothers was $36.6 \%$. Milk $301(33.7 \%)$ and plain water (24.4\%) were the most commonly pre-lacteal foods, 302 followed by sugar solution and butter. The most common reason to practice these 
pre-lacteal foods were cultural belief $(54.7 \%)$ and the desire to clean the new born's 304 throat and bowel (20.9\%) (Table 4).

Table 4: shows the magnitude of pre-lacteal foods and the reason to practice these foods in Dubti woreda, North East Ethiopia

Pre-lacteal foods and reason to practice them

Number

$(\%)$

\begin{tabular}{lll}
\hline Giving pre-lacteal foods & Yes & $86(36.6)$ \\
Types of pre-lacteal & No & $149(63.4)$ \\
foods given to the new born & Sugar solution & $21(24.4)$ \\
& Milk & $18(20.9)$ \\
Reason to feed these & Butter & $29(33.7)$ \\
pre-lacteal foods & Breast milk insufficient & $18(20.9)$ \\
& To clean new born throat and bowel & $11(12.8)$ \\
& Cultural belief & $47(54.7)$
\end{tabular}

307

Factors associated with pre-lacteal feeding practice in Dubti woreda, North East Ethiopia.

310 The binary logistic regression analysis revealed that those in the variables of 311 occupations, religion, mother's education, birth order of indexed child, gender of

312 indexed child, number of antenatal care visits, place of birth, breastfeeding initiation

313 time and breastfeeding counseling were significantly associated with pre-lacteal 314 feeding practice. When adjusting confounding factors, being a housewife (AOR=4.1, 
$95 \% \mathrm{Cl}: 1.4-12.2)$ and having male indexed child (AOR=4.9, 95\% $\mathrm{Cl}: 1.8-13.5)$ were more likely to practice pre-lacteal feeding than those of trade and female, respectively. However, pre-lacteal feeding practice was reduced by $69 \%$ in those mothers who had three and more antenatal care visits when compared to those mothers with less than three ante natal care visits ( $A O R=0.31,95 \% \mathrm{Cl}: 0.1-0.9)$. Mothers who give birth at health institutes had $90 \%$ lower practicing pre-lacteal feeding than those of home delivery $(A O R=0.1,95 \% \mathrm{Cl}: 0.02-0.3)$. Mothers who initiated breastfeeding within one hour had $94 \%$ lower pre-lacteal feeding practice than those who started breastfeeding after one day (AOR=0.04, at $95 \% \mathrm{Cl}: 0.01$ 0.1). Those who received breast feeding counseling were 0.21 times more likely to practice pre-lacteal feeding than those who did not $(\mathrm{AOR}=0.21$, at $95 \% \mathrm{Cl}: 0.06-0.7)$ (Table S1).

For qualitative data, a mother was selected purposively until redundant information was reached. A total of 17 participants were chosen based on this, with four of them serving as key informants. Ten and seven participants were selected from Algante and Dasa village, respectively. In each village, two key informants took part. Two of the four key informants were trained traditional birth attendants, whereas the other two were untrained traditional birth attendants. An in-depth interview was conducted with each of them. Participants were in the age range of $19-45$ years-old. The Muslim religious was followed by $82.4 \%$ (14/17) of participants, while the orthodox religion was followed by the remaining participants. About $88.2 \%(15 / 17)$ of study participants were housewives, while $11.8 \%$ were trades. Participants with no formal education were accounted for $82.4 \%$ and the rest of $17.6 \%$ had formal education. All of the mothers who had children under the age of two years gave birth at home. The key question we asked study participants concerning pre-lacteal foods was what you 
340 fed the new-born baby after delivery before starting breastfeeding. The pre-lacteal 341 foods found among study participants were milk product, some plant types, sweet 342 foods and water (Table 5).

343 Table 5: shows the theme, categories and codes of qualitative study result.

Theme Categories Codes

Pre-lacteal foods given Whater Spiritual water, plain water

to the new-born. Sweet foods Sugar solution, honey

Milk product Milk, butter

Plant type Dates (temir), condo pepper, leaf

345 The following study participants explained the above pre-lacteal feeding practice.

346 A 34-year-old mother expressed her practical observation on pre-lacteal feeding was 347 that "after birth, testing (the community called 'ono oru') was offered for my baby before supplying breast milk." when we asked what ono oru was given, she replied that "the new born must be given spiritual water (locally called 'zemzem water') 350 which came from Jidda", (an in-depth interview with mother).

351 According to a 30-year-old women explanation, "plain water, with sugar if available, 352 is used to clean the new-born's throat as well as serve as food. During my delivery, my breast did not secrete milk soon after birth. As a result, I gave sugar water for my baby as a food and to clean his throat", (in-depth interview with mother). 
A 38-years-old woman described that "mostly dates (locally called temir) and spiritual water (locally named as zemzem water) were offered for the new born. How do you give the new-born a date (temir)? She said that "first, dates (locally known as 'temir') was added in the water, and then I gave this dates (temir) water for the baby born within a short period because our religious ordered. This was passed down from elders to youths in our community" (in-depth interview with KII).

"After my baby was born, my mother gave for my baby camel milk (locally called 'han') before breast milk. Camel milk was usually given to babies since it was accessible and was thought to protect them from disease. Despite the fact that I did not feed honey to my infant; I saw also a number of mothers giving honey to their baby", a 25-year-old woman expressed, (in-depth interview with mother).

A 36-year-old mother explained, "I am a traditional birth attendant, and I gave milk, butter (locally known as 'sub ah'), or sugar water to the new born after delivery in our society. Butter is used to soften the baby's throat so that it can properly take breast milk the next time, whereas milk and sugar water are supplied as a food when breast milk is insufficient at the time of delivery. Currently, I am discontinuing this practice since training was given on the disadvantage of administering these food items prior to breastfeeding" (in-depth interview with KII).

A 37-year-old woman said that "Allah allowed to give spiritual water (locally called 'zemzem water') to the new born for keeping him healthy before giving breast milk, and my infant was also given spiritual water after delivery", (in-depth interview with mother).

A 35 years old mother reflected her practical observation after delivery was that "my child was given sugar water before breast-feeding. I have known that the majority of 
379 the mothers in our community were giving their children a small amount of either sugar water or butter because our religious ordered us to offer tests (locally called 'onu oru') for the newborn's post-delivery. Even though there was not sugar water available, just plain water was provided" (in-depth interview with mother).

A 33-year-old mother said that "my child was given dates (locally known as 'temir') water. She also pointed out that a leaf was given for the new born after birth to prevent fear and to be brave". What kind of leaf is it? We asked. She responded that "I did not know the leaf. Traditional elders recognized this, but they couldn't tell what kind of leaf it was", (in-depth interview with mother).

According to a 40-year-old mother, "my child was given spiritual water (locally called 'zemzem water') that came from Jidda. I've also heard from friends and others that a leaf given to a new baby helps him to be talkative, gentle, and hard during his adulthood time, and to take on the position of an elderly relative in the household. This leaf is secret; any one cannot know this leaf except the one already recognized. However, I did not give for my child this leaf', (in-depth interview with mother).

A 42-year-old woman assured regarding a hidden leaf, "A leaf was given for the babies that allow them to be community influential and to substitute his descend related hero place during adulthood period." Could you tell us what kind of leaf is it? No, I didn't tell. Community elders only recognize the leave, and exclusively give it to their families, relatives and close friends" (an in-depth interview with mother).

A 34-years mother described that "I have four children, and all of them were given 400 dates (locally known as 'temir') water after delivery. Dates (locally known as temir) 401 are considered important in our culture to prevent the child from several diseases. 
402 However, health education was now provided on the importance of exclusive 403 breastfeeding. So, we did not give it", (in-depth interview with mother).

404 A 19-year-old woman added that "since my breast did not secrete milk shortly after 405 birth, my child was given goat milk (locally called han). I know that most of my neighbors and relatives feed cow or goat milk their babies when their breasts don't produce milk", (in-depth interview with mother).

A 37-years old woman expressed her practice in the community was that "testing (locally known as onu oru) could be given for the new born as soon as delivery. This was previously a common practice in our community. The testing foods could be anything in the house, such as spiritual water, goat milk (locally known as hah), butter, or a sugar solution. As a result, I had provided similar things to my baby and others in the previous year. However, we no longer do so since health professionals have provided health education about the harmfulness of eating these foods before breast milk", (in-depth interview with KII).

A 25-year-old mother explained that "during delivery, the new born's throat was thought to be clogged with feces, fluids, or mucus. To ensure that the breast milk was adequately taken, plain water was provided to soften and clean the new born's throat prior to breastfeeding", (in-depth interview with mother).

32 year-old women also pointed out that "condo pepper (locally called kondo berbere) was given for my infant in the nose to be harsh and hero", (in-depth interview with mother).

423 Another 45-year-old woman asked about condo pepper and other foods 424 supplementation for new-born, and she said that "In the past, we used to give so 
425 many things to new-born in our community like condo pepper, leaf, and other foods.

426 However, the society does not have interest in giving these things because the

427 government and health professionals educated the society that giving these food 428 items can cause disease. Therefore, the practices of taking these foods were now 429 decreased. But, food items such as spiritual water (the local name is zemzem water), 430 dates (locally called temir) and milk are still frequently consumed after delivery" (in431 depth interview with KII).

432 A 33-years old mother explained that "when I gave birth to my child, I had honey in 433 my house, so I gave it to him. Honey is the preferred pre-lacteal meal and a better preventive mechanism against disease if it is present during delivery. But, honey was scarce in our neighborhood. As a result, milk and sugar water are often used as prelacteal foods in our neighborhood" (in-depth interview with mother). 
The purpose of the study was to determine the magnitude of pre-lacteal feeding practice and its influencing factors among women with children under the age of 2years in sub-districts of Dubti woreda, Afar region of Ethiopia. This study found that the important of different social-cultural and maternal health service utilization factors that are associated with pre-lacteal feeding practice, as well as types of pre-lacteal foods. In this study, the magnitude of pre-lacteal feeding was $36.6 \%$. This result is higher than that of a study in East Africa (3) and meta-analysis studies in Ethiopia (4, 11). Moreover, the present finding is higher than the previous studies in southern (5,

6) and northern part of Ethiopia $(8,9,15)$, as well as it supports a spatial and multilevel study in Ethiopia reported that the Afar region has the greatest rate of prelacteal feeding (10). The different between the current study and the previous studies could be attributed to regional and ethnic differences in traditional practices. The other variation may be health coverage disparities and a lack of effective health education between study settings. However, the magnitude of current finding is lower than that of study in south-west Ethiopia (7), Harare district in Eastern Ethiopia (12) and studies in Afar region (13,14). The variation could be study period, and study participants were near Samara, the Afar region's main city, where they might have access to maternal and child clinics, as well as health education or information via television and newspapers. Housewives were more likely to practice pre-lacteal feeding than those of trades. The finding is consistent with a study conducted in Afar region (13) and a meta-analysis study in Ethiopia (10). Mothers of male-indexed children were also more likely to practice pre-lacteal feeding than mothers of femaleindexed children. The reason could be that male children are given more attention in Afar society than female children (16), implying that gender inequality has an impact 
470 on pre-lacteal feeding practices. As a result, health education should be provided on

471 the equality of males with girls and the disadvantage of pre-lacteal feeding practice.

472 In the current study, pre-lacteal feeding practice was reduced by $69 \%$ in those 473 mothers who had three and more antenatal care visits when compared to those 474 mothers with less than three antenatal care visits. This evidence supports the 475 previous studies $(3,4,8,10,12)$. The reduction of pre-lacteal feeding could be that mothers have been given breastfeeding advice during antenatal care visits. Mothers who give birth at health institutes were less practicing pre-lacteal feeding than those of home delivery. This finding is in line with the previous studies $(3,4,6,7,10-12)$. The possible reason for this reduction of pre-lacteal feeding practice could be that the delivery was attended by health professionals who advised that breastfeeding should be started as soon as after birth. Those who received breast feeding counseling were $79 \%$ less likely to practice pre-lacteal feeding than those who did not. This is consistent with previous studies $(4-8,13)$. The decrease in pre-lacteal feeding may be attributed to better understanding of the benefits and harmfulness of pre-lacteal feeding practice during breastfeeding counseling. About $54 \%$ of mothers initiated breastfeeding within one hour. This result is lower than a study conducted in Afar region (15), but higher a previous study in this similar area (14). In this study, those who started breastfeeding within an hour had lower pre-lacteal feeding practice than those who started after one day. This evidence is similar to the previous studies $(1,4,7,9,12,13)$. This indicated that delayed breastfeeding caused pre-lacteal feeding practiced, which supports the finding of previous study in Sindh (1). Educational status of mothers and fathers had no statistically significant association with pre-lacteal feeding practice. This finding is against with the previous studies in the Southern (6) and North East region (13) of Ethiopia. The absence of 
difference in practicing pre-lacteal feeding between educated and uneducated participants could be owing to the influence of elders to undertake pre-lacteal feeding and giving high emphasis on cultural norms and beliefs in the community. The most common pre-lacteal foods mentioned by study participants were milk and plain water, followed by sugar solution and butter, respectively. These pre-lacteal foods were similar to the previous reported pre-lacteal foods that were studied in other areas $(2,5,12,13)$. Milk is commonly given as a pre-lacteal food because of the pastoral community in the study area. In the qualitative study, study participants reported further new information on pre-lacteal foods such as honey, dates (temir), a secret leaf, and condo pepper. Cultural views, the assumption cleaning new-born's throat and bowel, as well as breast milk insufficient were the most common reasons for using pre-lacteal foods. This finding is the same as that of the prior study in Sindh

This study's findings have a significant impact on the promotion of optimal breastfeeding practices and achieving sustainable development goal of decreasing child mortality in Ethiopia. The other strength of the current study is the inclusion of a qualitative study, which is used for triangulation and to gain additional information not found in the quantitative study. However, the information received from mothers could be subjected to recall bias, which was a limitation of this study.

516 Conclusion: In this study, pre-lacteal feeding practice is high. Those who have male indexed children and housewife were more likely to practice pre-lacteal feeding than those who have female indexed children and trades, respectively. However, mothers 519 who received three or more antenatal visits, delivery at a health institutes, 
520 breastfeeding initiation within one hour and getting breastfeeding counseling had

521 less pre-lacteal feeding practice. Pre-lacteal foods found in this study were milk

522 products, water, sweet foods, and some of plant types. The most common reasons

523 for practicing these food items were cultural beliefs and a desire to clean the new

524 born's throat and bowel.

526 List of abbreviation: KII: key informant interview, SPSS: social science statistical

527 package, AOR: adjusted odd ratio, MPH: master of public health.

\section{Authors' declaration}

529 Ethical approval and consent to participate: Ethical clearance was obtained from a research and ethics review committee of health science college, Samara

531 University. All methods were performed in accordance with the relevant guidelines and regulations. Informed consent was obtained from each study participant after explanation of the study's aim and from the legal guardians of patients with no formal

534 education. No one was harmed as a result of participating in this study. By eliminating any identifier from questionnaires, confidentiality was respected.

536 Consent for publication: Not applicable

537 Availability of data and material: All materials and data were in the manuscript and 538 supplementary materials (Table S1).

539 Competing interest: There is no any competing interest.

540 Funding: no funding source 
541 Acknowledgement: The authors like to thank all study participants and other

542 individuals involving in any process of this study.

543 Author contributions: Conceive and design the study: T.G.W, A.G and N.A,

544 Perform the study: T.G.W and N.A, Analysis the data: T.G.W, Wrote the paper: 545 T.G.W.

546

547

548

549

550

551

552

553

554

555

556

557

558

559 
561 1. Memon Y, Sheikh S, Memon A, Memon N. Feeding beliefs and practices of 562 mothers/caregivers for their infants. J Liaquat Uni Med Health Sci. 2006;5(1):8-13.

563 2. Shariff A, Farsana C. Breast feeding practices. Beliefs and taboos in 564 Karnataka. Journal of Social Work. 1990;51:143-8.

565 3. Birhan TY, Birhan NA, Alene M. Pooled Prevalence and Determinants of 566 Prelacteal Feeding Practice in Eastern Africa Evidence from Demographic and 567 Health Survey Data: A Multilevel Study. Risk management and healthcare policy. $568 \quad 2021 ; 14: 1085$.

569 4. Temesgen H, Negesse A, Woyraw W, Getaneh T, Yigizaw M. Prelacteal 570 feeding and associated factors in Ethiopia: systematic review and meta-analysis. 571 International breastfeeding journal. 2018;13(1):1-12.

572 5. Amele EA, wondimeneh Demissie B, Desta KW, Woldemariam EB. Prelacteal 573 feeding practice and its associated factors among mothers of children age less than 57424 months old in Southern Ethiopia. Italian journal of pediatrics. 2019;45(1):1-8.

575 6. Sorrie MB, Amaje E, Gebremeskel F. Pre-lacteal feeding practices and 576 associated factors among mothers of children aged less than 12 months in Jinka 577 Town, South Ethiopia, 2018/19. PloS one. 2020;15(10):e0240583.

578 7. Adem A, Assefa N, Deresa M, Yuya M, Ayana GM, Negash B, et al. 579 Prelacteal Feeding Practices and Its Associated Factors among Mother of Children 580 Less Than 2 Years of Age in Kersa District, Eastern Ethiopia. Global Pediatric $581 \quad$ Health. 2021;8:2333794X211018321.

582 8. Tekaly G, Kassa M, Belete T, Tasew H, Mariye T, Teshale T. Pre-lacteal 583 feeding practice and associated factors among mothers having children less than 
two years of age in Aksum town, Tigray, Ethiopia, 2017: a cross-sectional study. BMC pediatrics. 2018;18(1):1-10.

586

9. Gebremeskel SG, Gebru TT, Kassahun SS, Gebrehiwot BG. Magnitude of Prelacteal feeding and its associated factors among mothers having children less than one year of age: a community-based cross-sectional study in rural eastern zone, Tigray, Ethiopia. Advances in Public Health. 2020;2020.

10. Teshale AB, Worku MG, Tesema GA. Spatial distribution and determinants of the change in pre-lacteal feeding practice over time in Ethiopia: A spatial and multivariate decomposition analysis. PloS one. 2021;16(1):e0244574.

11. Takele WW, Tariku A, Wagnew F, Ekubagewargies DT, Getinet W, Derseh L, et al. Magnitude of prelacteal feeding practice and its association with place of birth in Ethiopia: a systematic review and meta-analysis, 2017. Archives of Public Health. 2018;76(1):1-11.

12. Bekele $\mathrm{Y}$, Mengistie B, Mesfine F. Prelacteal feeding practice and associated factors among mothers attending immunization clinic in Harari region public health facilities, Eastern Ethiopia. Open Journal of Preventive Medicine. 2014;2014.

13. Liben ML, Wuneh AG, Zepro NB, Mulugeta A. Factors associated with prelacteal feeding in Afar regional state, northeastern Ethiopia: a cross sectional study. International Journal of Research-Granthaalayah. 2017;5(7):116-27.

14. Hussien J, Assefa S, Liben ML. Breastfeeding performance in Afar regional 604 state, northeastern Ethiopia: a cross sectional study. BMC pediatrics. 2018;18(1):1605 8.

15. Liben ML, Gemechu YB, Adugnew M, Asrade A, Adamie B, Gebremedin E, et 607 al. Factors associated with exclusive breastfeeding practices among mothers in dubti 
608 town, afar regional state, Northeast Ethiopia: a community based cross-sectional 609 study. International breastfeeding journal. 2016;11(1):1-6.

610 16. Reda KT. Social organization and cultural institutions of the Afar of Northern 611 Ethiopia. International Journal of Sociology and Anthropology. 2011;3(11):423-9.

612 17. Andarge MY. The Difficulties of Ending Female Genital Mutilation (FGM):

613 Case of Afar Pastoralist Communities in Ethiopia. Retrieved fromwww ohchr 614 org/Documents/Issues/Women/WRGS/FGM/NGOs/ActionFor. 2014. 


\section{Supplementary Files}

This is a list of supplementary files associated with this preprint. Click to download.

- SupplementarytabletableS1.docx 